\title{
Development and internal validation of risk prediction model of metabolic syndrome in oil workers
}

Jie Wang ${ }^{1}$, Chao Li ${ }^{1}$, Jing Li ${ }^{1}$, Sheng Qin' ${ }^{1}$ Chunlei Liư ${ }^{3}$, Jiaojiao Wang ${ }^{1}$, Zhe Chen ${ }^{1}$, Jianhui $\mathrm{Wu}^{1,2^{*}}$ and Guoli Wang ${ }^{1,2}$

\begin{abstract}
Background: The prevalence of metabolic syndrome continues to rise sharply worldwide, seriously threatening people's health. The optimal model can be used to identify people at high risk of metabolic syndrome as early as possible, to predict their risk, and to persuade them to change their adverse lifestyle so as to slow down and reduce the incidence of metabolic syndrome.

Methods: Design existing circumstances research. A total of 1468 workers from an oil company who participated in occupational health physical examination from April 2017 to October 2018 were included in this study. We established the Logistic regression model, the random forest model and the convolutional neural network model, and compared the prediction performance of the models according to the F1 score, sensitivity, accuracy and other indicators of the three models.

Results: The results showed that the accuracy of the three models was $82.49,95.98$ and $92.03 \%$, the sensitivity was $87.94,95.52$ and $90.59 \%$, the specificity was $74.54,96.65$ and $94.14 \%$, the F1 score was $0.86,0.97$ and 0.93 , and the area under ROC curve was $0.88,0.96$ and 0.92 , respectively. The Brier score of the three models was $0.15,0.08$ and 0.12 , Observed-expected ratio was 0.83, 0.97 and 1.13, and the Integrated Calibration Index was 0.075,0.073 and 0.074, respectively, and explained how the random forest model was used for individual disease risk score.

Conclusions: The study showed that the prediction performance of random forest model is better than other models, and the model has higher application value, which can better predict the risk of metabolic syndrome in oil workers, and provide corresponding theoretical basis for the health management of oil workers.
\end{abstract}

Keywords: Data mining, Oil workers, Metabolic syndrome, Risk prediction

\footnotetext{
* Correspondence: wujianhui555@163.com

${ }^{1}$ School of Public Health, North China University of Science and Technology, No.21 Bohai Avenue, Caofeidian New Town, Tangshan City, Hebei Province 063210, P.R. China

${ }^{2}$ Hebei Province Key Laboratory of Occupational Health and Safety for Coal

Industry, North China University of Science and Technology, Tangshan,

Hebei, P.R. China

Full list of author information is available at the end of the article
}

(c) The Author(s). 2020 Open Access This article is licensed under a Creative Commons Attribution 4.0 International License, which permits use, sharing, adaptation, distribution and reproduction in any medium or format, as long as you give appropriate credit to the original author(s) and the source, provide a link to the Creative Commons licence, and indicate if changes were made. The images or other third party material in this article are included in the article's Creative Commons licence, unless indicated otherwise in a credit line to the material. If material is not included in the article's Creative Commons licence and your intended use is not permitted by statutory regulation or exceeds the permitted use, you will need to obtain permission directly from the copyright holder. To view a copy of this licence, visit http://creativecommons.org/licenses/by/4.0/. The Creative Commons Public Domain Dedication waiver (http://creativecommons.org/publicdomain/zero/1.0/) applies to the data made available in this article, unless otherwise stated in a credit line to the data. 


\section{Background}

Metabolic syndrome (MetS) refers to the accumulation of multiple metabolic risk factors in the body including obesity, impaired glucose regulation, dyslipidemia and hypertension. MetS is a group of complex clinical syndromes based on insulin resistance. Relevant literatures have shown that metabolic syndrome increases the risk of cardiovascular disease, type 2 diabetes and chronic kidney disease [1-3].With the social and economic development and changes in people's lifestyles, the prevalence of metabolic syndrome has increased year by year and brought a heavy economic burden, which has become an important health issue of common concern to people worldwide.

At present, the definition and diagnostic criteria of metabolic syndrome have not been completely unified. In 1998, WHO officially named the "metabolic syndrome" and and proposed corresponding diagnostic criteria for the first time [4].Over the course of the next decade, the diagnostic criteria for metabolic syndrome have undergone many changes and revisions, including 2001 national cholesterol education program adult treatment group report for the third time (NCEP ATP III). Chinese diabetes association (CDS) diagnostic criteria in 2004. International diabetes federation (IDF) diagnostic criteria 2005. In 2009, the American heart association (AHA), the international diabetes federation, the national heart, lung and blood institute and other institutions jointly proposed a tentative unified standard [58].According to a large number of epidemiological data, the global prevalence of MetS is about $30 \%$ [9]. DoosupShin based on 2007-2014 national health and nutrition survey data on MetS prevalence statistics found that American adults MetS prevalence rate has reached 34.3\% (according to the revised NCEP-ATP III diagnostic criteria) [10].In South Korea, according to the same diagnostic criteria, the prevalence rate of metabolic syndrome in adults from 2009 to 2013 was as high as 30.52 \%[11].In China, in 2010, Jieli L u[12] and others conducted a data report analysis of 97,098 adults in China, and estimated the prevalence of MetS was 33.9\% (according to the NCEP-ATP III diagnostic criteria).In 2015, Ting Liu analyzed the prevalence of MetS among 34,025 residents in Jilin province and found that the prevalence of MetS was $32.5 \%$ (according to IDF diagnostic criteria) [13].In 2016, Ri L i[14] and others conducted a meta-analysis showing that the prevalence of MetS in subjects over 15 years old was $24.5 \%$ (according to IDF diagnostic criteria).Although the diagnostic criteria are not uniform, it is undeniable that metabolic syndrome has become one of the chronic diseases with high incidence in China and even in the world.

Data mining refers to extracting hidden information and knowledge with potential research value from large data, which is often used in the medical field with large amounts of data and fast update speed. Among them, the classification algorithm has been widely concerned and applied in recent years. This algorithm takes a variety of risk factors affecting the occurrence of disease as a prerequisite, and uses statistical methods and computer algorithms to build a predictive model of disease risk. The constructed model is used to predict the probability of a certain population or individual developing a certain disease, and then provides a theoretical basis for personal health management and corresponding preventive measure $s[15]$.At present, Logistic regression, Cox regression, BP neural network, decision tree, support vector machine and other models are mostly used to construct metabolic syndrome risk models at home and abroad [16-18]. These models can be used to identify high-risk groups of MetS, persuade them to change their unhealthy lifestyles, reduce and slow down the occurrence and development of the disease. Among the $\mathrm{m}[19-21]$, Logistic regression and Cox regression, as traditional statistical modeling methods, are widely used and have strong explanatory power. However, Cox regression is often used for survival analysis data, which requires two dependent variables at the same time and has relatively strict requirements on data. The decisionmaking tree model has strong visibility, but is prone to overfitting and poor generalization effect. The random forest model is a classifier composed of multiple decision-making trees, which improves the weak generalization ability of a single decision-making tree and balances the error of unbalanced data. As a kind of artificial neural network model, BP neural network is fault-tolerant to some extent, but local minimization problems often occur, and the learning speed is slow, and the phenomenon of overfitting is easy to occur. In the convolutional neural network model, the local receptive field and weight sharing of convolutional kernel reduce the computational complexity and have high accuracy and good generalization ability. Due to regional and cultural differences, the effects of existing models vary, and mature and accurate metabolic syndrome risk prediction systems have not been established at home and abroad. Moreover, most of these models established at present are aimed at the assessment of the risk of disease in the general population, ignoring the special group of occupational population.

As an important part of China's non-renewable energy industry, the petroleum industry still accounts for a large proportion in the national economy. Oil workers are also the main laborers in the production of the secondary industry in China. Their health will affect the development of China's economy to a certain extent and should be paid more attention. Oil workers are affected by high temperature, noise, shift work and other harmful 
occupational factors, as well as a variety of adverse lifestyles caused by occupational stress, which can greatly increase the incidence of metabolic syndrome to some extent. For special occupational group, the risk prediction model of ordinary people is no longer suitable for them, so it is necessary to establish a risk prediction model of metabolic syndrome for them, so as to achieve early detection, diagnosis and treatment, and protect the health of oil workers. In this study, a certain oil industry worker was selected as the research object, and the traditional Logistic regression model, random forest model and the recent thermal convolutional neural network model were developed and internally verified. The prediction performance of each model is compared to find the optimal model, which provides a theoretical basis for the health management of this special occupation group of oil workers.

\section{Methods}

\section{Data sources and research objects}

This study strictly followed the Transparent Reporting of a Multivariable Prediction Model for Individual Prognosis or Diagnosis (TRIPOD) reporting guidelines and prepared this report according to the relevant guideline $s[22]$. This study adopted the existing circumstances research method. In order to avoid the contingency caused by manual partition of the data set and make the sample more representative, this study adopted 10 -fold cross validation (The data set was divided into 10 mutually exclusive subsets on average. The union of 9 subsets was taken as the training set each time, and the remaining 1 subset was the test set.The cycle was repeated 10 times to represent the final performance of the model by the average of all test results) for data partition and internal verification, so as to further improve the robustness of model prediction.

A total of 1468 workers from an oil company who attended occupational examination and physical examination from April 2017 to October 2018 were selected as the research objects. Inclusion criteria: length of service 1 year or above. Aged between 18 and 60. Complete questionnaire and physical examination data. All subjects gave their informed consent for inclusion before they participated in the study. The study was conducted in accordance with the Declaration of Helsinki, and the protocol was approved by the Ethics Committee of North China University of Science and Technology(NO.16040).

\section{Outcomes and predictor variables}

One-to-one questionnaire survey was conducted on oil workers by uniformly trained personnel to collect the following information: (1) General situation: gender, age, education, income status, marital status, etc. (2) Lifestyle: smoking, drinking, diet and physical exercise. (3) history of personal and family diseases: hyperglycemia, hypertension, hyperlipidemia, etc. (4)Working conditions: shifts, exposure to high temperature, noise and other harmful factors. (5)Physical examination: height, weight, blood pressure and waist circumference, etc.

The study subjects took venous blood in the early morning after fasting for $12 \mathrm{~h}$, and tested the biochemical indicators such as fasting blood glucose, highdensity lipoprotein, and triglyceride using the Dirion CS1200 automatic biochemical analyzer (China Changchun Dirion Medical Technology Company). The diagnostic criteria of metabolic syndrome [8] can be diagnosed if it meets three or more of the following five indicators:

I. Central obesity: Chinese people have a waist circumference $\geq 85 \mathrm{~cm} \quad$ (male). waist circumference $\geq 80 \mathrm{~cm}$ (female).

II. Elevated blood glucose: FBG $\geq 5.6 \mathrm{mmol} / \mathrm{L}$ or those who have been diagnosed with diabetes and receive treatment.

III. TG $\geq 1.7 \mathrm{mmol} / \mathrm{L}$ or those who have been diagnosed with hypertriglyceridemia and received treatment.

IV. HDL-C $<1.04 \mathrm{mmol} / \mathrm{L}$ (male). HDL-C $<1.30$ $\mathrm{mmol} / \mathrm{L}$ (female) or those who have been diagnosed with low-density lipoproteinemia and received treatment.

V. Systolic / diastolic blood pressure $\geq 130 / 85 \mathrm{mmHg}$ or those diagnosed with hypertension and receiving treatment.

\section{Quality control}

The investigators can only take up their posts after unified training. The collected questionnaire data are collected on the spot for double and double check and input, and the questionnaires with incorrect input are checked for the third time to ensure the accuracy of the collected data. The same instrument was used for physical examination and laboratory test, and the biochemical indicators were tested by the same kit in North China Petroleum Underground Hospital.

\section{Sample size}

Through consulting a large number of relevant literatures, it was found that there were about 15 predictive factors related to metabolic syndrome.General neural network and random forest model require that the sample content is more than 2 times of explanatory variables.The newly developed Logistic regression model $R^{2}$ CS_adj (the estimated measure after adjusting the overfitting of the model) is at least 0.1 , so to achieve the expected contraction coefficient of 0.9 [23], we finally need a sample size of at least 1274 .

\section{Statistical methods}

CscrMainUI system developed by a scientific research company was used to scan and input questionnaires and 
establish a database. IBM SPSS19.0 was used for statistical analysis. The measurement data obeying the normal distribution were expressed as $\overline{\mathrm{x}} \pm \mathrm{s}$, and the $\mathrm{t}$ test was used for comparison between groups. The non-normally distributed measurement data were represented by $[M$ (P25,P75)], and the rank sum test was used for comparison between groups. The count data were used as the ratio, and Pearson $x^{2}$ test was used for comparison between groups. Unconditional binary classification logistic regression was used for multivariate analysis. The independent variable introduction criterion was $P \leq 0.05$, and the test level $\alpha=0.05$ (both sides).

\section{Establishment and validation of the models}

Input variables of the three models: predictors of metabolic syndrome of oil workers determined by multivariate logistic regression analysis and results of a large number of relevant literature reviews. The output variable was whether metabolic syndrome occurred.

First of all, logistic regression model, random forest model and convolutional neural network model were respectively constructed by Python's Numpy. Use regularization techniques for the three models respectively (that is, by increasing the training error and reducing the test error to constrain the parameters to be optimized) to prevent over-fitting. After that, the data set was divided into 10 parts, one part as test set and the other nine parts as training set. The three training models were created by using the functions of Python Sklearn library to train the training set, and the test data were used for model evaluation and prediction. Finally, the average of ten operations was taken as the true index of the model. During the establishment of logistic regression model, the corresponding function of Sklearn library was called to establish the training model. For the construction of random forest, in order to ensure the robustness of the model, the Shuffle function in sklearn library was used to disrupt the data set. In order to ensure the randomness of data selection, the Gini coefficient was used as the classification index to predict the model. In the construction of the convolutional neural network model, the input characteristics were first standardized, $x^{*}=\frac{x-\min }{\max -\min }$, the original data was normalized to the interval $[0,1]$, and two pooling layers and two full connection layers were used. The size of the output activation was calculated according to the size of the input activation (W), the size of the receiving domain of the convolutional layer neurons (F), the step size they apply (S), and the size of the zero fill used on the boundary $(\mathrm{P})$. The formula was $(\mathrm{W}-\mathrm{F}+2 \mathrm{P}) / \mathrm{S}+1$. The relu function $\mathrm{f}(x)=$ $\max (0, x)$ was used as the excitation function to further reduce the error, and the maximum pooling was used in the pooling process. Finally, the matplotlib library was used to visualize the three models respectively and calculate the confusion matrix of the results. The internal validation of the model was carried out by 10 -fold cross-validation, and the sensitivity, specificity, F1 score, area under ROC curve, Brier score, observed-expected ratio and other indicators of the three models were compared.

\section{Results}

\section{General situation}

Of the 1468 oil workers, 1105 were male, with an average age of 43(38,48),363 were women, with an average age of $44(42,47)$. The prevalence rate of metabolic syndrome in petroleum workers was $40.67 \%$, among which, the rate of central obesity was $56.81 \%$, the rate of abnormal blood glucose was $49.39 \%$, the rate of abnormal triglyceride was $32.90 \%$, the rate of abnormal HDL was $19.28 \%$, and the rate of abnormal blood pressure was 55.99\%. As shown in Fig.1.

\section{Independent variable screening}

Single factor analyses were performed on the basic conditions, diet and lifestyle, occupational exposure factors and laboratory tests of 1468 oil workers. The results showed statistically significant differences in age, gender, Body Mass Index(BMI), marital status, family history of hypertension, family history of diabetes mellitus, salt, meat intake, smoking status, drinking status, shift work situation, Occupational heat, noise, hemoglobin, uric acid(UA), alanine transaminase(ALT), etc. $(P<0.05)$, are shown in Tables 1, 2, 3 and 4.

The significant factors of univariate analysis were included in the multivariate nonconditional Logistic regression analysis. The results showed that the risk of metabolic syndrome increased with age, BMI, UA and ALT. People with a family history of diabetes, a strong salt taste, occasional consumption of dairy products, daily consumption of carbonated beverages, smoking, shift work, and exposure to high temperatures are more likely to develop metabolic syndrome. The protective factors of metabolic syndrome include family income of 2000-3000 yuan per capita, daily consumption of dairy products and physical exercise. Combined with the results of relevant literature review, 13 significant factors in the multivariate analysis were taken as independent variables for the establishment of the model, as shown in Tables 5 and 6.

\section{Collinearity diagnosis}

The diagnosis of collinearity was made by using the binary correlation coefficient $r$, tolerance and variance inflation factor(VIF).The results showed that the correlation coefficient $|\mathrm{r}|$ was 0.31 at most and $|\mathrm{r}|<0.5$, as shown in Supplementary Table 1, Additional File 1. The minimum tolerance was 0.844 , much higher than 0.1 , and the maximum variance inflation factor was 1.185, less than 5, as shown in Supplementary Table 


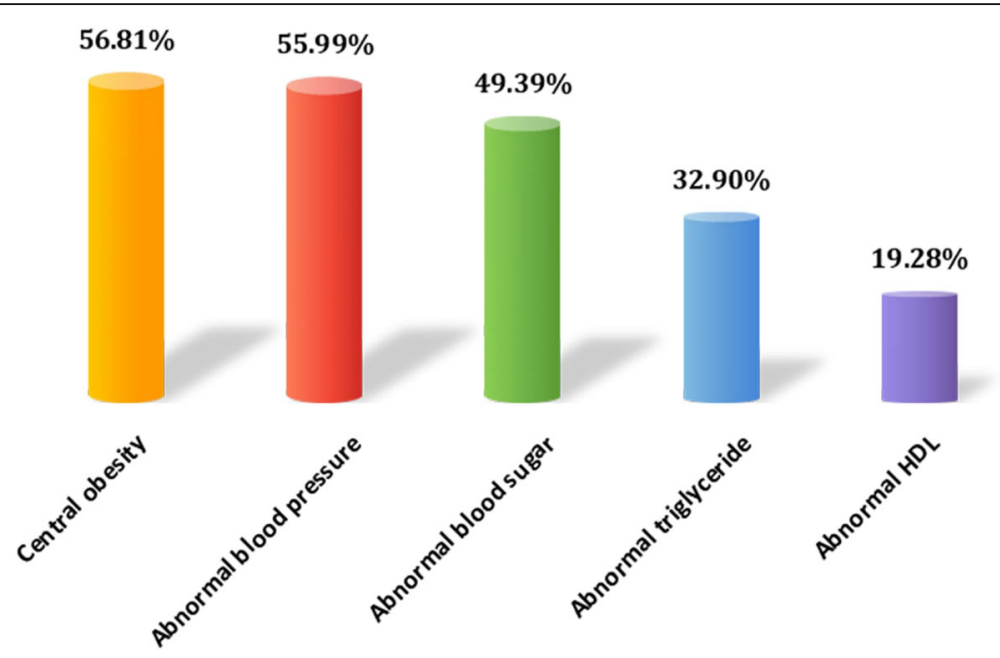

Fig. 1 Comparison of abnormal rates among components of metabolic syndrome

2, Additional File 2. The above results indicate that there is no serious multicollinearity among the screened independent variables.

\section{Evaluation of model results}

In this study, the sample data were divided by the 10fold cross-validation method. Three models, namely Logistic Regression, Random Forest and $\mathrm{CNN}$, were established respectively to learn and predict the data set. The prediction results of each model were compared with the actual results of the sample, so as to obtain the respective confusion matrix of the model, as shown in Table 7. Among the three models, age, ALT, BMI and UA all rank in the top four in terms of the importance of predictive variables, as shown in Supplementary Figure 1-3, Additional Files 3, 4 and 5.

Table 1 Comparison of the basic conditions of oil workers with and without metabolic syndrome

\begin{tabular}{|c|c|c|c|c|c|}
\hline \multirow[t]{2}{*}{ Basic conditions } & \multirow[t]{2}{*}{ Category(Unit) } & \multicolumn{2}{|c|}{ MetS n(\%)/M(P $\left(P_{25}, P_{75}\right)$} & \multirow[t]{2}{*}{$x^{2} / Z$} & \multirow[t]{2}{*}{$P$} \\
\hline & & No & Yes & & \\
\hline Age & Year & $43(38,47)$ & $44(40,49)$ & -5.79 & $<0.001$ \\
\hline \multirow[t]{2}{*}{ Gender } & Male & $601(69.00)$ & $504(84.42)$ & 45.26 & $<0.001$ \\
\hline & Female & $270(31.00)$ & $93(15.58)$ & & \\
\hline BMI & $\mathrm{Kg} / \mathrm{m}^{2}$ & $23.9(21.90,25.90)$ & $26.80(24.90,28.80)$ & -16.35 & $<0.001$ \\
\hline \multirow[t]{3}{*}{ Marital status } & Unmarried & $56(6.43)$ & $15(2.51)$ & 11.82 & 0.003 \\
\hline & Married & $782(89.78)$ & 559(93.63) & & \\
\hline & Others & $33(3.79)$ & 23(3.85) & & \\
\hline \multirow[t]{3}{*}{ Education level } & Junior high school and below & 133(15.27) & 104(17.42) & 9.07 & 0.011 \\
\hline & High school/technical secondary school & $374(42.94)$ & $290(48.58)$ & & \\
\hline & College and above & $364(41.79)$ & 203(34.00) & & \\
\hline \multirow[t]{3}{*}{ Per capita monthly household income(Yuan) } & $<2000$ & 619(71.07) & $454(76.05)$ & 8.05 & 0.018 \\
\hline & $2000 \sim$ & $212(24.34)$ & 109(18.26) & & \\
\hline & $3000 \sim$ & $40(4.59)$ & $34(5.70)$ & & \\
\hline \multirow[t]{2}{*}{ Family history of hypertension } & No & $489(56.14)$ & $288(48.24)$ & 8.88 & 0.003 \\
\hline & Yes & $382(43.86)$ & $309(51.76)$ & & \\
\hline \multirow[t]{2}{*}{ Family history of hyperlipidemia } & No & $801(91.96)$ & $538(90.12)$ & 1.51 & 0.22 \\
\hline & Yes & $70(8.04)$ & 59(9.88) & & \\
\hline \multirow[t]{2}{*}{ Family history of diabetes mellitus } & No & $725(83.24)$ & $454(76.05)$ & 11.58 & 0.001 \\
\hline & Yes & 146(16.76) & 143(23.95) & & \\
\hline
\end{tabular}


Table 2 Comparison of diet and lifestyle of oil workers with and without metabolic syndrome

\begin{tabular}{|c|c|c|c|c|c|}
\hline \multirow[t]{2}{*}{ Factors } & \multirow[t]{2}{*}{ Category } & \multicolumn{2}{|c|}{ MetS $n(\%) / M\left(P_{25}, P_{75}\right)$} & \multirow[t]{2}{*}{$x^{2}$} & \multirow[t]{2}{*}{$P$} \\
\hline & & No & Yes & & \\
\hline \multirow[t]{3}{*}{ Salt } & Light & $221(25.37)$ & $88(14.74)$ & 26.39 & $<0.001$ \\
\hline & Moderate & $381(43.74)$ & $276(46.23)$ & & \\
\hline & Salty & $269(30.88)$ & 233(39.03) & & \\
\hline \multirow[t]{4}{*}{ Meat intake } & Never & $23(2.64)$ & 13(2.18) & 9.38 & 0.025 \\
\hline & Occasionally & 198(22.73) & $101(16.92)$ & & \\
\hline & Regularly & $335(38.46)$ & $232(38.86)$ & & \\
\hline & Every day & $315(36.17)$ & $251(42.04)$ & & \\
\hline \multirow[t]{4}{*}{ Fruit intake } & Never & $37(4.25)$ & $27(4.52)$ & 6.59 & 0.086 \\
\hline & Occasionally & 278(31.92) & $223(37.35)$ & & \\
\hline & Regularly & $258(29.62)$ & $146(24.46)$ & & \\
\hline & Every day & 298(34.21) & $201(33.67)$ & & \\
\hline \multirow[t]{4}{*}{ Dairy intake } & Never & $127(14.58)$ & 103(17.25) & 119.81 & $<0.001$ \\
\hline & Occasionally & $230(26.41)$ & $297(49.75)$ & & \\
\hline & Regularly & 199(22.85) & $111(18.59)$ & & \\
\hline & Every day & $315(36.17)$ & $86(14.41)$ & & \\
\hline \multirow[t]{4}{*}{ Carbonated beverage intake } & Never & $370(42.48)$ & $270(45.23)$ & 10.52 & 0.015 \\
\hline & Occasionally & $384(44.09)$ & $258(43.22)$ & & \\
\hline & Regularly & 79(9.07) & $31(5.19)$ & & \\
\hline & Every day & $38(4.36)$ & $38(6.37)$ & & \\
\hline \multirow[t]{2}{*}{ Physical exercise } & No & $307(35.25)$ & 259(43.38) & 9.90 & 0.002 \\
\hline & Yes & $564(64.75)$ & $338(56.62)$ & & \\
\hline \multirow[t]{3}{*}{ Smoking status } & No smoking & $524(60.16)$ & $262(43.89)$ & 39.30 & $<0.001$ \\
\hline & Quit smoking & $51(5.86)$ & $61(10.22)$ & & \\
\hline & Smoking & 296(33.98) & $274(45.90)$ & & \\
\hline \multirow[t]{3}{*}{ Drinking status } & No drinking & $585(67.16)$ & $309(51.76)$ & 37.02 & $<0.001$ \\
\hline & Alcohol withdrawal & $16(1.84)$ & $24(4.02)$ & & \\
\hline & Drinking & $270(31.00)$ & $264(44.22)$ & & \\
\hline
\end{tabular}

Table 3 Comparison of occupational exposure factors of oil workers with and without metabolic syndrome

\begin{tabular}{|c|c|c|c|c|c|}
\hline \multirow[t]{2}{*}{ Factors } & \multirow[t]{2}{*}{ Category } & \multicolumn{2}{|c|}{ MetS n(\%)/M(P $\left.\mathrm{P}_{25}, \mathrm{P}_{75}\right)$} & \multirow[t]{2}{*}{$x^{2}$} & \multirow[t]{2}{*}{$P$} \\
\hline & & No & Yes & & \\
\hline \multirow[t]{3}{*}{ Shift work situation } & Never & $535(61.42)$ & $254(42.55)$ & 51.44 & $<0.001$ \\
\hline & Once & 208(23.88) & 202(33.84) & & \\
\hline & Now & $128(14.70)$ & $141(23.62)$ & & \\
\hline \multirow[t]{3}{*}{ Labour intensity } & Mild & $93(10.68)$ & $44(7.37)$ & 5.36 & 0.069 \\
\hline & Moderate & 434(49.83) & 295(49.41) & & \\
\hline & Severe & 344(39.49) & $258(43.22)$ & & \\
\hline \multirow[t]{2}{*}{ Occupational heat } & No & $548(62.92)$ & $266(44.56)$ & 48.34 & $<0.001$ \\
\hline & Yes & $323(37.08)$ & $331(55.44)$ & & \\
\hline \multirow[t]{2}{*}{ Noise } & No & $429(49.25)$ & $206(34.51)$ & 31.39 & $<0.001$ \\
\hline & Yes & $442(50.75)$ & $391(65.49)$ & & \\
\hline
\end{tabular}

The accuracy of three models, Logistic Regression Model, Random Forest Model and CNN, was 82.49, 95.98 and $92.03 \%$, respectively. The sensitivity was $87.94,95.52$ and $90.59 \%$, respectively. The specificity was 74.54, 96.65 and $93.10 \%$, respectively. F1 Score was $0.86,0.97$ and 0.93 respectively. The area under ROC curve was $0.88,0.96$ and 0.92 , respectively. The Brier score of the three models was $0.15,0.08,0.12$, observed-expected ratio was $0.83,0.97,1.13$, real-inthe large was $0.109,0.099,0.098$, ICI was 0.075 , $0.073,0.074$, respectively. The calibration diagrams of logistic regression model, random forest model and CNN model were all close to the diagonal, and there was no serious deviation from the calibration results. The random forest model performs better than Logistic Regression model and CNN in both discrimination and calibration. See Table 8 and Fig.2. 
Table 4 Comparison of laboratory tests in oil workers with and without metabolic syndrome

\begin{tabular}{|c|c|c|c|c|}
\hline \multirow{2}{*}{$\begin{array}{l}\text { Biochemical } \\
\text { Indicators }\end{array}$} & \multicolumn{2}{|l|}{ MetS n(\%)/M(P $\left.P_{25}, P_{75}\right)$} & \multirow[t]{2}{*}{$z$} & \multirow[t]{2}{*}{$P$} \\
\hline & No & Yes & & \\
\hline $\mathrm{RBC}\left(\times 10^{12} / \mathrm{L}\right)$ & $5.01(4.65,5.33)$ & $5.29(4.99,5.54)$ & -6.94 & $<0.001$ \\
\hline$M C V(f l)$ & $88.80(85.10,92.00)$ & $88.20(84.80,91.80)$ & -0.85 & 0.397 \\
\hline $\mathrm{BPC}\left(\times 10^{12} / \mathrm{L}\right)$ & $256.00(219.50,290.75)$ & $251.00(211.00,284.00)$ & -0.55 & 0.59 \\
\hline $\mathrm{MPV}(\mathrm{fl})$ & $8.20(7.70,8.80)$ & $8.20(7.70,8.80)$ & -0.83 & 0.405 \\
\hline Hemoglobin(g/L) & $155(141,165)$ & $160(151,169)$ & -6.44 & $<0.001$ \\
\hline TBIL(mmol/L) & $13.50(10.50,17.70)$ & $13.45(10.30,17.10)$ & -0.81 & 0.421 \\
\hline $\mathrm{UA}(\mathrm{mmol} / \mathrm{L})$ & $307(242,373)$ & $367(304,426)$ & -11.13 & $<0.001$ \\
\hline $\mathrm{ALT}(\mathrm{U} / \mathrm{L})$ & $20.00(14.00,24.00)$ & $35.00(21.00,45.00)$ & -17.07 & $<0.001$ \\
\hline
\end{tabular}

Random forest model for individual risk score calculation The random forest risk prediction model established in this study was the optimal model suitable for risk prediction of metabolic syndrome in oil workers. To further apply the model to reality and calculate the individual risk score, predict_proba method of the Sklearn library in Python can be used. The dependent variable $\mathrm{Y}$ is whether the person has metabolic syndrome, and the independent variable $\mathrm{X}$ is the 13 predictive factors in this study, and then the risk score of a certain person or a group of people can be obtained. The higher the score, the greater the risk of the disease. As shown in Table 6, Code IV, Additional File 6.

\section{Discussion}

At present, all countries in the world have recognized that the establishment of disease risk prediction model has a greater role in preventing and controlling the occurrence of metabolic syndrome, and established the corresponding MetS model based on the epidemiological data. In 2008, Fabien Szabo DE Edelenyi et al. in France conducted a large case-control study and found that the prediction accuracy of metabolic syndrome status using random forest classification technique was $71.70 \%(72.10 \%$ in the control group and $70.70 \%$ in the case group) [24].In 2010, Lin CC in Taiwan established an artificial neural network model and a Logistic regression model to identify metabolic syndrome in 383 patients with schizophrenia, and the results showed that the accuracy was 88.30 and $83.60 \%$, the sensitivity was 93.10 and $86.20 \%$, and the specificity was 86.90 and $83.80 \%$, respectively [25]. In 2015, Worachartcheewa $\mathrm{n}[26]$ et al. used the random forest model to establish a prediction model of metabolic syndrome for 5646 adults living in Bangkok, and the accuracy was 98.11\%.In 2016,

Table 5 Multivariate nonconditional Logistic regression analysis of influencing factors in oil workers with metabolic syndrome

\begin{tabular}{|c|c|c|c|c|c|c|}
\hline Factors & $B$ & S.E & Wald $x^{2}$ & $P$ & OR & $95 \% \mathrm{Cl}$ \\
\hline Age & 0.088 & 0.012 & 55.251 & 0.000 & 1.092 & $1.067,1.118$ \\
\hline Per capita monthly household income(2000 ) & -0.77 & 0.22 & 12.244 & 0.000 & 0.463 & $0.301,0.713$ \\
\hline Per capita monthly household income(3000 ) & 0.166 & 0.388 & 0.184 & 0.668 & 1.181 & $0.552,2.525$ \\
\hline $\mathrm{BMI}$ & 0.273 & 0.026 & 114.091 & 0.000 & 1.313 & $1.249,1.381$ \\
\hline Family history of diabetes mellitus & 0.373 & 0.183 & 4.129 & 0.042 & 1.452 & $1.013,2.080$ \\
\hline Salt(Moderate) & 0.86 & 0.206 & 17.429 & 0.000 & 2.362 & $1.578,3.536$ \\
\hline Salt(Salty) & 0.555 & 0.214 & 6.759 & 0.009 & 1.742 & $1.146,2.648$ \\
\hline Dairy intake(Occasionally) & 0.676 & 0.216 & 9.771 & 0.002 & 1.966 & $1.287,3.003$ \\
\hline Dairy intake(Every day) & -1.149 & 0.261 & 19.317 & 0.000 & 0.317 & $0.190,0.529$ \\
\hline Carbonated beverage intake(Every day) & 1.102 & 0.365 & 9.148 & 0.002 & 3.012 & $1.474,6.153$ \\
\hline Physical exercise & -0.398 & 0.152 & 6.86 & 0.009 & 0.672 & $0.499,0.905$ \\
\hline Smoking status(Smoking) & 0.431 & 0.181 & 5.675 & 0.017 & 1.539 & $1.079,2.194$ \\
\hline Shift work situation(Once) & 0.974 & 0.172 & 32.184 & 0.000 & 2.648 & $1.892,3.707$ \\
\hline Shift work situation(Now) & 1.509 & 0.237 & 40.489 & 0.000 & 4.522 & $2.841,7.198$ \\
\hline Occupational heat & 0.656 & 0.224 & 8.548 & 0.003 & 1.926 & $1.241,2.989$ \\
\hline UA & 0.004 & 0.001 & 27.244 & 0.000 & 1.004 & $1.003,1.006$ \\
\hline ALT & 0.029 & 0.005 & 40.946 & 0.000 & 1.030 & $1.020,1.039$ \\
\hline
\end{tabular}


Table 6 Assignment of influencing factor variables

\begin{tabular}{lll}
\hline Variable name & Variable meaning & Assignment method \\
\hline$Y$ & MetS & $0=$ No, $1=$ Yes \\
$X_{1}$ & Age & Continuous variable (year) \\
$X_{2}$ & Per capita monthly household income & $1=<2000,2=2000-3000,3=\geq 3000$ \\
$X_{3}$ & BMl & Continuous variable(Kg/m2) \\
$X_{4}$ & Family history of diabetes mellitus & $1=$ No,2=Yes \\
$X_{5}$ & Salt & $1=$ Light,2= Moderate,3=Salty \\
$X_{6}$ & Dairy intake & $1=$ Never,2=Occasionally,3=Regularly,4=Every day \\
$X_{7}$ & Carbonated beverage intake & $1=$ Never,2=Occasionally,3=Regularly,4=Every day \\
$X_{8}$ & Physical exercise & $1=$ No,2=Yes \\
$X_{9}$ & Smoking status & $1=$ No smoking,2=Quit smoking,3=Smoking \\
$X_{10}$ & Shift work situation & $1=$ Never,2=Once,3=Now \\
$X_{11}$ & Occupational heat & $1=$ No,2=Yes \\
$X_{12}$ & UA & Continuous variable(mmol/L) \\
$X_{13}$ & ALT & Continuous variable(U/L) \\
\hline
\end{tabular}

karimi-alavijeh et al. used 2107 participants in the Iranian cohort study to establish the decision-making tree model and support vector machine model, and found that the accuracy was 73.90 and $75.70 \%$, the sensitivity was 75.80 and $77.40 \%$, and the specificity was 72.00 and $74.00 \%[27]$.The established models have local applicability advantages due to the differences in region, population and input variables.

The results of this study showed that the prevalence of MetS in workers of an oil company was $40.67 \%$, higher than the average level of Chinese adults [12-14].At the same time, the prevalence rate of the five diagnostic criteria of metabolic syndrome ranged from high to low, which were: central obesity, abnormal blood pressure, abnormal blood glucose, abnormal triglyceride, and abnormal high-density lipoprotein. The occurrence of this

Table 7 Sample classification results of Logistic regression model, Random Forest model and Convolutional neural network model [N (\%)]

\begin{tabular}{cllll}
\hline \multirow{2}{*}{ Model } & predictive & \multicolumn{2}{l}{ Actual value } & \\
\cline { 3 - 4 } & value & Yes & No & Total \\
\hline Logistic regression model & Yes & $766(87.94)$ & $152(25.46)$ & 918 \\
& No & $105(12.06)$ & $445(74.54)$ & 550 \\
& Total & 871 & 597 & 1468 \\
Random forest model & Yes & $832(95.52)$ & $20(3.35)$ & 852 \\
& No & $39(4.48)$ & $577(96.65)$ & 616 \\
& Total & 871 & 597 & 1468 \\
CNN & Yes & $789(90.59)$ & $35(5.86)$ & 824 \\
& No & $82(9.41)$ & $562(94.14)$ & 644 \\
& Total & 871 & 597 & 1468 \\
\hline
\end{tabular}

phenomenon was related to the generally good living conditions, dietary habits, irregular life and rest oil workers. According to the importance of predictive variables in the three models established, it was found that the top four variables were age, ALT, BMI and UA, indicating that these four factors played a very important role in the development of metabolic syndrome.In the process of independent variable screening, age, income, BMI, family history of diabetes, salt intake, physical exercise and other factors were the influencing factors of metabolic syndrome, which was consistent with previous results $[28,29]$. UA and ALT were found to be risk factors for MetS, and related studies showed that UA increased the risk of MetS by increasing insulin resistance, and increased ALT in the blood might cause fat accumulation in the liver. Through investigation, Mandana

Table 8 Comparison of predictive performance of the three models

\begin{tabular}{llll}
\hline Evaluation index & $\begin{array}{l}\text { Logistic } \\
\text { regression } \\
\text { model }\end{array}$ & $\begin{array}{l}\text { Random } \\
\text { forest } \\
\text { model }\end{array}$ & CNN \\
\hline Accuracy rate(\%) & 82.49 & 95.98 & 92.03 \\
Sensitivity(\%) & 87.94 & 95.52 & 90.59 \\
Specificity(\%) & 74.54 & 96.65 & 94.14 \\
F1 Score & 0.86 & 0.97 & 0.93 \\
AUC & 0.88 & 0.96 & 0.92 \\
Brier score & 0.15 & 0.08 & 0.12 \\
observed-expected ratio & 0.83 & 0.97 & 1.13 \\
calibration-in-the-large & 0.109 & 0.099 & 0.098 \\
Integrated Calibration Index & 0.075 & 0.073 & 0.074 \\
\hline
\end{tabular}



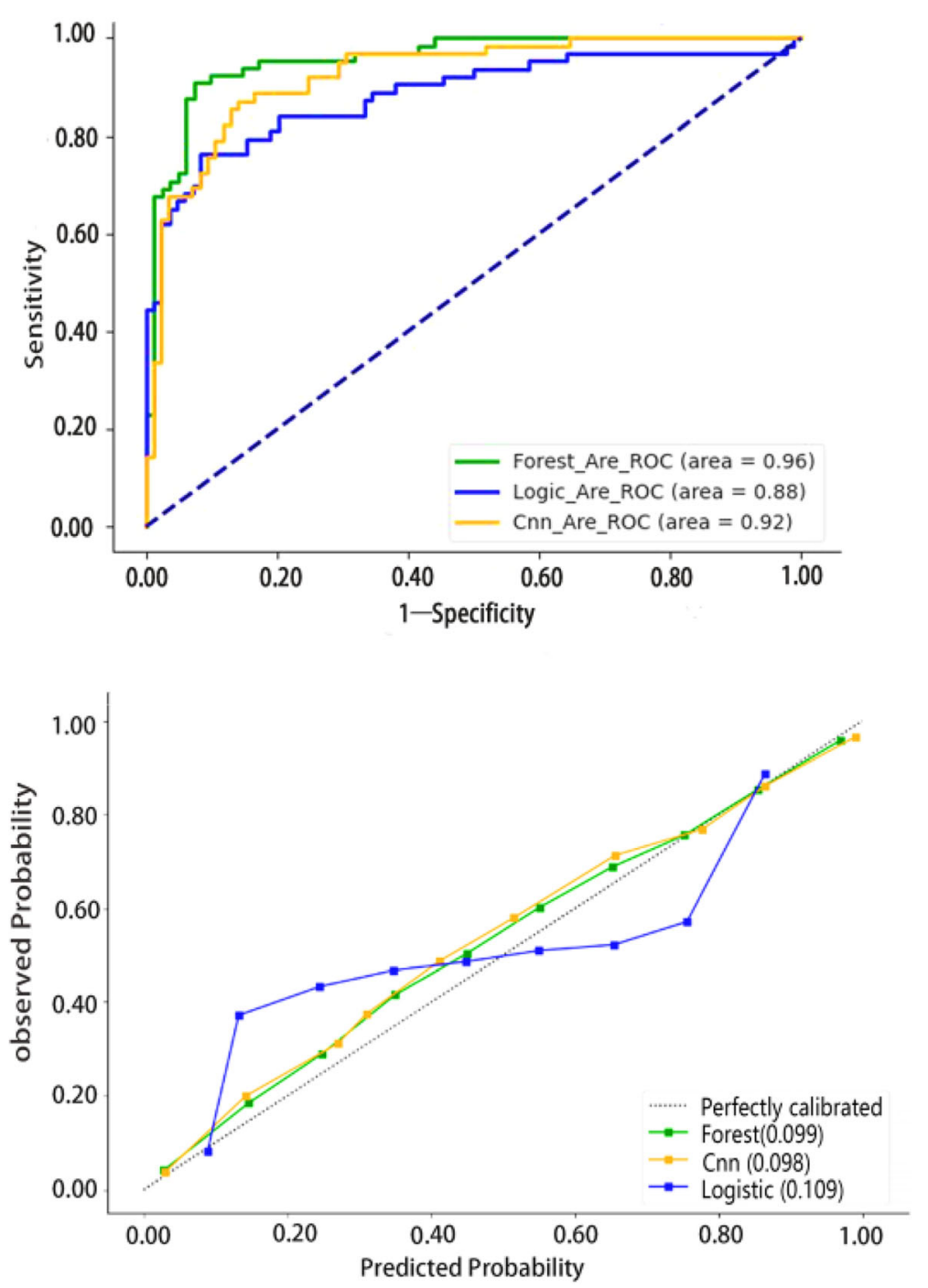

Fig. 2 ROC curves and calibration curves of three predictive models

Khalili et al. found that patients with MetS had higher hepatic steatosis level, and there was a correlation between the elevation of ALT and MetS [30, 31].Different from the general population, oil workers have been in a special occupational environment for a long time. High temperature environment causes the body's circulatory system to be in a long-term stress state, resulting in decreased elasticity of blood vessel wall, increased blood viscosity, and increased blood pressure. In addition, studies have shown that high temperature contact can affect insulin hemodynamics, resulting in insulin resistance in the body [32, 33].Harmony between biological rhythm and natural rhythm is the basis of normal physiological activities. Irregular shift work will affect the biological rhythm of human body due to irregular circadian rhythm, resulting in the disturbance of nutrients and related hormones in the body, thus resulting in glucose and lipid metabolism disorder and energy imbalance [34].On the other hand, the workers of night shift work lack of sleep time, and the incidence of unhealthy lifestyle such as smoking, drinking and irregular diet increases greatly, which are the driving forces for the occurrence of metabolic syndrome [35].

In this study, Logistic regression model, random forest model and convolutional neural network model were established to compare their prediction performance. In this study, it was found that the random forest model had higher discriminance and calibration, and was more suitable for the risk prediction of metabolic syndrome of oil workers. As a prediction model for the risk of metabolic syndrome in petroleum workers, the model with higher discrimination is more suitable for the early detection of patients, so as to play a real role in early detection, early diagnosis 
and early treatment of the disease, namely secondary prevention of the disease. A good clinical disease risk prediction model should not only have good discrimination, but also consider whether it is well calibrated. In this study, Brier Score, O/E ratio, calibration-inthe-large, and Integrated Calibration Index (ICI)were also introduced to evaluate the calibration degree of the model. Among them, the IC I[36] refers to the weighted average of the absolute average difference between the observed probability and the predicted probability, and can be used to quantify the calibration method in the results of dichotomization, so as to evaluate the calibration effect more comprehensively. As an emerging machine learning algorithm in recent years, random forest mode $1[37,38]$ is a highly flexible classifier containing multiple decision trees. The random forest model solves the shortcoming of the decision tree algorithm, and adopts the random sampling method to enhance the generalization ability. Proposed by Yann Lecun of New York university in 1988, the convolutional neural network model is the first truly successful deep learning method using multi-layer hierarchical network, including input layer, hidden layer (convolutional layer, pooling layer, full connection layer) and output layer, which effectively reduces the number of network parameters and significantly reduces the computational complexity. Previously, convolutional neural network was mainly used for image, language and medical imaging processing. In recent years, it has also been used as a neural network model to predict the risk of various diseases [39-41].However, the prediction effect of $\mathrm{CNN}$ for different diseases is uneven, which may be because the model construction needs to be further improved and there is no unified standard yet. At the same time, a certain amount of data is required for model training. Logistic regression model is a traditional statistical modeling method, which is widely used in the field of risk factor screening and disease prediction. It is convenient to use and the meaning of the parameters is clear, but it cannot solve the nonlinear problems and the application conditions are strict. The sample size increases with the increase of input variables, and the predictive power decreases when the data do not meet the requirements [42].

Due to the limitation of research conditions, this study has certain limitations. This paper only developed and internally validated the metabolic syndrome risk prediction model for oil workers, and did not conduct external validation of the model. The choice of model input variables will directly affect the prediction effect of the model, which needs to be further explored.This study was based on a cross-sectional study.Only the prevalence data of metabolic syndrome of oil workers were available, and the causal relationship between the prevalence and predictive factors could not be determined.

\section{Conclusions}

Three risk prediction models (Logistic regression model, random forest model and convolutional neural network model) for the occurrence of metabolic syndrome in petroleum workers were established and compared. The results show that the random forest model has better discriminant degree and calibration degree, and has higher robustness. It shows that the random forest model can predict the risk of metabolic syndrome in oil workers more accurately, and can provide health education for high-risk employees with metabolic syndrome and put forward corresponding prevention strategies, so as to improve the allocation of national medical and health resources and the distribution of health services.

\section{Supplementary Information}

The online version contains supplementary material available at https://doi. org/10.1186/s12889-020-09921-w.

Additional file 1: Supplementary Table 1. Coefficient of correlation. Additional file 2: Supplementary Table 2. Results of tolerance and variance inflation factor.

Additional file 3: Supplementary Figure 1. The importance of predictive variables in Logistic regression model.

Additional file 4: Supplementary Figure 2. The importance of predictive variables in random forest model.

Additional file 5: Supplementary Figure 3. The importance of predictive variables in CNN.

Additional file 6. I: Logistic regression model code. II: CNN code. III: Random forest model code. IV: Risk score code.

\section{Abbreviations}

MetS: Metabolic Syndrome; WHO: World Health Organization; NCEP ATP III: National Cholesterol Education Program Adult Treatment group report for the third time; CDS: Chinese Diabetes association; IDF: International Diabetes Federation; AHA: American Heart Association; BMI: Body Mass Index;

RBC: Red Blood Cell; MCV: Erythrocyte Mean Corpuscular Volume; BPC: Blood Platelet Count; MPV: Mean Platelet Volume; UA: Uric Acid; ALT: Alanine transaminase; OR: Odds ratio; 95\%Cl: 95\% Confident limit; SE: Standard Error; VIF: Variance Inflation Factor; CNN: Convolution Neural Network; AUC: Area Under the Curve; ICl: Integrated Calibration Index; O/E ratio: Observedexpected ratio

\section{Acknowledgements}

We would like to thank north China university of science and technology for providing a software and hardware platform and financial support to ensure the smooth progress of this research.We would also like to thank our teachers and classmates for their help and warmth in the research process.

\section{Authors' contributions}

Design research, J.W. and J.H.W.; Methodology, C.L.L., Z.C. and G.L.W.; Project administration, C.L., J.L., S.Q. and J.J.W.; Software, J.W. and C.L.L.; Validation, J.H.W. and G.L.W.; Writing original draft, J.W.; Writing review, J.W. and J.H.W..All authors responded to the modification of the study protocol and approved the final manuscript. 


\section{Funding}

This work was supported by the National Key R\&D Program of China (No.2016YFC0900605).The funding body had no role in the study design and collection, analysis, and interpretation of data and in writing the manuscript.

\section{Availability of data and materials}

The data that support the findings of this study are available from [Institute of basic medicine, Chinese academy of medical sciences] but restrictions apply to the availability of these data, which were used under license for the current study, and so are not publicly available. Data are however available from the authors upon reasonable request and with permission of [Institute of basic medicine, Chinese academy of medical sciences].

\section{Ethics approval and consent to participate}

All procedures performed in studies involving human participants were in accordance with the ethical standards of the institutional and/or national research committee and with the 1964 Helsinki declaration and its later amendments or comparable ethical standards. The study was approved by the Ethics Committee of North China University of Science and Technology(NO.16040).All individuals in the study signed a paper version of the informed consent.

\section{Consent for publication}

Not applicable.

\section{Competing interests}

The authors declare that they have no competing interests.

\section{Author details}

${ }^{1}$ School of Public Health, North China University of Science and Technology, No.21 Bohai Avenue, Caofeidian New Town, Tangshan City, Hebei Province 063210, P.R. China. ${ }^{2}$ Hebei Province Key Laboratory of Occupational Health and Safety for Coal Industry, North China University of Science and Technology, Tangshan, Hebei, P.R. China. ${ }^{3}$ College of Science, North China University of Science and Technology, Tangshan, Hebei, P.R. China.

\section{Received: 22 May 2020 Accepted: 18 November 2020}

\section{Published online: 30 November 2020}

\section{References}

1. Li W, Song F, Wang $X$, et al. Relationship between metabolic syndrome and its components and cardiovascular disease in middle-aged and elderly Chinese population: a national cross-sectional survey. BMJ Open. 2019;9(8): e27545.

2. Low S, Khoo K, Wang J, et al. Development of a metabolic syndrome severity score and its association with incident diabetes in an Asian population-results from a longitudinal cohort in Singapore. Endocrine. 2019;65(1):73-80.

3. Chen J, Kong X, Jia X, et al. Association between metabolic syndrome and chronic kidney disease in a Chinese urban population. Clin Chim Acta. 2017; 470:103-8.

4. Alberti KG, Zimmet PZ. Definition, diagnosis and classification of diabetes mellitus and its complications. Part 1: diagnosis and classification of diabetes mellitus. Provisional report of a WHO consultation. Diabet Med. 1998;15(7):539-53.

5. Kuhar MB. Executive summary of the third report of the National Cholesterol Education Program (NCEP) expert panel on detection, evaluation, and treatment of high blood cholesterol in adults (adult treatment panel III). Circulation. 2001;106(25):2486-97.

6. Metabolic Syndrome Research Group of Diabetes Branch of Chinese Medical Association. Recommendations of the Chinese Medical Association diabetes branch on metabolic syndrome. Chin J Diabetes. 2004;12(3):156-61.

7. Alberti KG, Zimmet P, Shaw J. Metabolic syndrome-a new world-wide definition.A consensus statement from the international diabetes federation. Diabetic Med. 2006;23(5):469-80.

8. Alberti $\mathrm{K}$, Eckel RH, Grundy SM, et al. Harmonizing the metabolic syndrome: a joint interim statement of the international diabetes federation task force on epidemiology and prevention; national heart, lung, and blood institute; American heart association; world heart federation; international atherosclerosis society; and international association for the study of obesity. Circulation. 2009;120(16):1640-5.

9. Al-Thani MH, Al-Thani AAM, Cheema S, et al. Prevalence and determinants of metabolic syndrome in Qatar: results from a National Health Survey. BMJ Open. 2016;6(9):e9514.

10. Shin D, Kongpakpaisarn K, Bohra C. Trends in the prevalence of metabolic syndrome and its components in the United States 2007-2014. Int J Cardiol. 2018;259:216-9.

11. Lee $S E$, Han K, Kang YM, et al. Trends in the prevalence of metabolic syndrome and its components in South Korea: findings from the Korean National Health Insurance Service database (2009-2013). PLoS One. 2018; 13(3):e194490.

12. Lu J, Wang L, Li M, et al. Metabolic syndrome among adults in China: the 2010 China noncommunicable disease surveillance. J Clin Endocrinol Metab. 2017;102(2):507-15.

13. Liu T. Prevalence and risk factors of metabolic syndrome among residents in Jilin Province. MA dissertation. Jilin: Jilin University; 2017.

14. Li R, Li W, Lun Z, et al. Prevalence of metabolic syndrome in mainland China: a meta-analysis of published studies. BMC Public Health. 2016;16(1):296-306.

15. Li QZ, Rui Z. Research progress on evaluation methods of fit degree of disease risk prediction model. Chin Health Stat. 2015;32(3):544-6.

16. Choe EK, Rhee H, Lee $\mathrm{S}$, et al. Metabolic syndrome prediction using machine learning models with genetic and clinical information from a nonobese healthy population. Genom Inform. 2018;16(4):e31.

17. Worachartcheewan A, Schaduangrat N, Prachayasittikul V, et al. Data mining for the identification of metabolic syndrome status. EXCLI J. 2018;17:72-88.

18. Mu DY, Hu W, Ma Y, et al. Influencing factors and risk forecast model of metabolic syndrome among college faculties, Chengdu. Modern Prev Med. 2019;46(1):36-42.

19. Fatekurohman M, Nurmala N, Anggraeni D. Comparison of exact, efron and breslow parameter approach method on hazard ratio and stratified cox regression model. J Phys Conf Ser. 2018;1008(1):e012007.

20. Sohrabi S, Atashi A, Dadashi A, et al. A comparative study of multilayer neural network and C4. 5 decision tree models for predicting the risk of breast Cancer. Archiv Breast Cancer. 2018;5(1):11-4.

21. Tran DP, Hoang VD. Adaptive learning based on tracking and Reldentifying objects using convolutional neural network. Neural Process Lett. 2019;50(1): 263-82.

22. Gary SC, Johannes BR, Douglas GA, et al. Transparent reporting of a multivariable prediction model for individual prognosis or diagnosis (TRIPOD): the TRIPOD statement. Eur Urol. 2015:67(6):1142-51.

23. Riley RD, Snell Kl, Ensor J, et al. Minimum sample size for developing a multivariable prediction model: PART ॥ - binary and time-to-event outcomes. Stat Med. 2019:38(7):1276-96.

24. Szabo DEF, Goumidi L, Bertrais S, et al. Prediction of the metabolic syndrome status based on dietary and genetic parameters, using random Forest. Genes Nutr. 2008;3(3):173-6.

25. Lin CC, Bai YM, Chen JY, et al. Easy and low-cost identification of metabolic syndrome in patients treated with second-generation antipsychotics: artificial neural network and logistic regression models. J Clin Psychiatry. 2010;71(3):225-34.

26. Worachartcheewan A, Shoombuatong W, Pidetcha P, et al. Predicting metabolic syndrome using the random forest method. Sci World J. 2015. https://doi.org/10.1155/2015/581501.

27. Karimi-Alavijeh F, Jalili S, Sadeghi M. Predicting metabolic syndrome using decision tree and support vector machine methods. ARYA Atherosclerosis. 2016;12(3):146-52

28. Soltani S, Moslehi N, Hosseini-Esfahani F, et al. The association between empirical dietary inflammatory pattern and metabolic phenotypes in overweight/obese adults. Int J Endocrinol Metab. 2018;16(2):e60048.

29. Antonella A, Andrea M, Sarka K, et al. Association of Dietary Patterns with metabolic syndrome: results from the Kardiovize Brno 2030 study. Nutrients. 2018;10(7):898-914.

30. Rashidi H, Shahbazian H, Nokhostin F, et al. The comparison of insulin and uric acid levels in adolescents with and without metabolic syndrome. Front Biol. 2018;13(6):452-7.

31. Khalili M, Shuhart MC, Lombardero M, et al. Relationship between metabolic syndrome, alanine aminotransferase levels, and liver disease severity in a multiethnic north American cohort with chronic hepatitis B. Diabetes Care. 2018;41(6):1251-9. 
32. James SM, Honn KA, Gaddameedhi S, et al. Shift work: disrupted circadian rhythms and sleep-implications for health and well-being. Curr Sleep Med Rep. 2017;3(2):104-12.

33. Vinogradova I, Anisimov V. Melatonin prevents the development of the metabolic syndrome in male rats exposed to different light/dark regimens. Biogerontology. 2013;14(4):401-9.

34. Schwartsburd PM. Catabolic and anabolic faces of insulin resistance and their disorders: a new insight into circadian control of metabolic disorders leading to diabetes. Future Science OA. 2017;3(3):1-10.

35. Kar D, Gillies C, Nath M, et al. Association of smoking and cardiometabolic parameters with albuminuria in people with type 2 diabetes mellitus: a systematic review and meta-analysis. Acta Diabetol. 2019;56(8):839-50.

36. Peter CA, Ewout WS. The integrated calibration index (ICI) and related metrics for quantifying the calibration of logistic regression models. Stat Med. 2019:38:4051-65.

37. Al-Quraishi T, Abawajy JH, Chowdhury MU, et al. Breast Cancer recurrence prediction using random Forest model. Recent Adv Soft Comput Data Mining. 2018;700:318-29

38. Dagliati A, Marini S, Sacchi $L$, et al. Machine learning methods to predict diabetes complications. J Diabetes Sci Technol. 2018;12(2):295-302.

39. Wu JH, Li J, Wang J, et al. Risk prediction of type 2 diabetes in steel workers based on convolutional neural network. Neural Comput \& Applic. 2020; 32(3):9683-98

40. Ševo I, Avramović A. Convolutional neural network based automatic object detection on aerial images. IEEE Geosci Remote Sens Lett. 2016;13(5):740-4.

41. Mu-han D. Prediction of epileptic seizures based on convolution neural network. MA dissertation. Shandong: Shandong Normal University; 2018.

42. Zhang $M$, Wang $L M$, Chen $\mathrm{ZH}$, et al. Multilevel logistic regression analysis on hypercholesterolemia related risk factors among adults in China. Chin J Prev Med. 2018;52(2):151-7.

\section{Publisher's Note}

Springer Nature remains neutral with regard to jurisdictional claims in published maps and institutional affiliations.

Ready to submit your research? Choose BMC and benefit from:

- fast, convenient online submission

- thorough peer review by experienced researchers in your field

- rapid publication on acceptance

- support for research data, including large and complex data types

- gold Open Access which fosters wider collaboration and increased citations

- maximum visibility for your research: over $100 \mathrm{M}$ website views per year

At $\mathrm{BMC}$, research is always in progress.

Learn more biomedcentral.com/submissions 